\title{
Diagnostic performance of GI-RADS reporting system in evaluation of adnexal masses
}

\author{
Sahar Mahmoud Abd elsalam*, Soha Talaat Hamed² and Mohamed Abd elghafar Sayed ${ }^{3}$
}

\begin{abstract}
Background: Transvaginal and pelvic ultrasound are considered the primary imaging modality in evaluating adnexal masses. Gynaecologic Imaging Reporting and Data System (GI-RADS) depends on different ultrasound patterns and criteria adopted by the International Ovarian Tumour Analysis (IOTA) group. The current study aimed to detect the diagnostic accuracy of the GI-RADS classification in evaluating adnexal masses. In this prospective cross-sectional study, a total of 112 adnexal masses belonging to 100 women, age ranged 12 to 66 years old, were included. The study population was recruited throughout the period between January and November 2017. Ultrasound examination was performed to all patients; different US and Doppler criteria were assessed.

Results: Out of the 112 lesions, 36 (32.1\%) were GI-RADS 2, 32 (28.6\%) GI-RADS 3, 13 (11.6\%) GI-RADS 4, and 31 (27.7\%) GI-RADS 5. The GI-RADS classification showed sensitivity $97 \%$, specificity $84.8 \%$, positive predictive value (PPV) 72.7\%, negative predictive value (NPV) 98.5\%, and accuracy $88.4 \%$.

Conclusion: The GI-RADS reporting system carried a high sensitivity in identifying adnexal masses at high risk of malignancy. The increased number of benign lesions misclassified as GI-RADS 4 required additional markers to improve the specificity in GI-RADS classification.
\end{abstract}

Keywords: Gl-RADS, Adnexal masses, Sensitivity, Benign, Malignant ovarian masses

\section{Background}

Adnexal masses are considered common gynaecologic clinical problems. Most lesions are benign necessitating conservative management and follow-up $[1,2]$. Ovarian cancer is considered the most lethal gynaecological cancer in women. Annually, it is responsible for an estimated 152,000 deaths, and 239,000 new cases are recorded worldwide [3]. In Egypt, it accounts for 4.1\% of all cancers affecting women, considering it one of the most common diagnosed cancers among the Egyptian females [4]. Typically, ovarian cancer presents at late stage when its 5-year survival rate is less than $30 \%$ [5].

\footnotetext{
*Correspondence: shahdsahar@yahoo.com; saharmahmoud575@gmail.com 'Department of Radiology, Faculty of Medicine, Beni-Suef University, Beni Suef, Egypt

Full list of author information is available at the end of the article
}

Adnexal masses are identified primarily by ultrasound [6]. However, since the ultrasound is operator-dependent and the diagnosis of adnexal masses has been usually left to the examiners' impression, many scoring systems, regression models, and neural networks have been suggested for better diagnosis [7-11].

In 2009, Amor and colleagues proposed the Gynaecology Imaging Reporting and Data System (GI-RADS, Table 1) [12], to enhance the communication between radiologists and clinicians. This classification is based on summarized standardized report of ultrasound findings which could provide an estimated risk of malignancy for the examined adnexal mass [13].

Accurate pre-operative assessment of women with adnexal masses is crucial for ovarian reserve in case of nonmalignant pathologies particularly in young fertile women [14]. Also, the precise determination of the characteristics 
Table 1 GI-RADS classification system for adnexal masses [11]

\begin{tabular}{|c|c|c|c|}
\hline $\begin{array}{l}\text { GIRADS } \\
\text { grade }\end{array}$ & Diagnosis & $\begin{array}{l}\text { Est. prob. } \\
\text { malignancy }\end{array}$ & Details \\
\hline 1 & $\begin{array}{l}\text { Definitive } \\
\text { benign }\end{array}$ & $0 \%$ & Normal ovaries identified, and no adnexal mass seen \\
\hline 2 & $\begin{array}{l}\text { Very probably } \\
\text { benign }\end{array}$ & $<1 \%$ & Adnexal lesions thought to be of functional origin, e.g. follicles, corpora lutea, hemorrhagic cysts \\
\hline 3 & Probably benign & $1-4 \%$ & $\begin{array}{l}\text { Neoplastic adnexal lesions thought to be benign, such as endometrioma, teratoma, simple cyst, } \\
\text { hydrosalpinx, paraovarian cyst, peritoneal pseudocyst, pedunculated myoma, or findings suggestive of } \\
\text { pelvic inflammatory disease }\end{array}$ \\
\hline 4 & $\begin{array}{l}\text { Probably } \\
\text { malignant }\end{array}$ & $5-20 \%$ & $\begin{array}{l}\text { Any adnexal lesion not included in GI-RADS } 1-3 \text { and with one or two findings suggestive of } \\
\text { malignancy* }\end{array}$ \\
\hline 5 & $\begin{array}{l}\text { Very probably } \\
\text { malignant }\end{array}$ & $>20 \%$ & Adnexal masses with three or more findings suggestive of malignancy* \\
\hline
\end{tabular}

The asterisk denotes findings suggestive of malignancy included thick papillary projections, thick septa, solid areas with/without ascites and vascularization within solid areas

of the adnexal masses is important in cases when laparoscopy replaces laparotomy because aseptic oncologic methods have to be followed to prevent rupture of adnexal malignant masses [15]. Further, pre-operative suspicion of ovarian cancer enables the examiners to do another imaging modality for proper characterization and staging of the lesions as well as improvement of survival rates [16].

In this regard, the aim of our study was to determine the diagnostic performance of GI-RADS reporting system in the evaluation of adnexal masses and decreased inconclusive ultrasound results.

\section{Methods}

\section{Patients}

Our observational cross-sectional study included 100 female patients who were attending the outpatient clinics of Obstetrics and Gynaecology and referred to radiology department of University hospital. The study was conducted between January and November 2017.

The study was conducted in full accordance with the guidelines for Good Clinical Practice and the Declaration of Helsinki, and data for patients were collected only after obtaining their informed written consents.

The participating women were asked to fill-in a questionnaire including questions about their age, complaints, and obstetric history. All women were assessed clinically by general and local pelvic examination.

\section{Methods and techniques}

Abdominal and transvaginal ultrasound examinations were done for all cases (excluding one patient, aged 12 years virgin, who was evaluated by abdominal ultrasound only). The ultrasound examination was done by one of the two experienced radiologists (10 and 20 years of experience). We used Toshiba Xario 200 and voluson E6 devices to perform the ultrasound examination. Transabdominal scan using a $3.5-5-\mathrm{MHz}$ sector transducer was done.
The examination required filling of the urinary bladder (ideal $1-2 \mathrm{~cm}$ above the uterine fundus). Images were obtained in sagittal and transverse planes (oblique image may be needed). To view the adnexa, we moved the transducer from side to side. Transvaginal sonography (TVS) using a $4-8-\mathrm{MHz}$ endoluminal probe after emptying the urinary bladder to minimize discomfort and to bring the uterus and ovaries into the focal zone was performed. The probe was disinfected, ultrasound (US) gel was applied to the transducer head, and a condom was used. Anteroposterior and transverse pelvic planes were done. Colour and power Doppler were done for all cases to detect the vascularity of the lesions and to differentiate between suspicious solid component and benign lesions.

\section{Image interpretation}

We assessed the morphological and colour Doppler findings of the lesions. The morphological criteria included the site of the lesion, size, the echopattern, the presence of associated solid component, and the presence of septa or papillary projections. The colour Doppler was used to detect the vascularity, high or low, and vessel arrangement, central or peripheral. Absent or mild peripheral vascularity was considered benign; however, abnormal central vascularity of the solid component was considered suspicious lesions.

We used GI-RADS classification system for adnexal masses, in which GI-RADS 1 was considered definitely benign, GI-RADS 2 very probably benign, GI-RADS 3 probably benign, GI-RADS 4 probably malignant, and GI-RADS 5 very probably malignant (Table 1) [12].

Findings suggestive of malignancy included thick papillary projections, thick septa, solid areas with/without ascites and vascularization within solid areas, papillary projections or central area of a solid tumour on colour or power Doppler assessment as defined according to the International Ovarian Tumour Analysis criteria [11]. According to the GI-RADS classification system, 
management protocol is offered to the gynaecologist as shown in Fig. 1.

Histopathologic diagnosis was the gold standard in patients managed by surgical treatment (63 lesions in our study). The other 49 lesions showed resolution and improvement on follow-up ultrasound scans.

\section{Statistical analysis}

Data entry, verification, and validation were carried out using standard computer software. Data were analysed using the software, Statistical Package for Social Science (SPSS Inc. Released 2009, PASW Statistics for Windows, version 18.0: SPSS Inc., Chicago, IL, USA) then processed and tabulated.

Frequency distribution with its percentage and descriptive statistics with mean and standard deviation were calculated. Chi-square and $t$ test were done whenever needed. $P$ values of less than 0.05 were considered significant. The sensitivity, specificity, positive predictive value (PPV), negative predictive value (NPV), and accuracy of the GI-RADS system were based on considering GI-RADS 2 and 3 as benign and GI-RADS 4 and 5 as high risk of malignancy.

\section{Results}

A total of 100 women with 112 adnexal masses were included in this study. Patients were complaining of pelvic pain, pelvic masses, and/or menstrual irregularities. Patient's mean age was $37.9 \pm 12.9$ (ranging from 12 to 66 years). Premenopausal patients were 82 cases; 71 cases
(86.5\%) revealed benign pathology, and 11 cases (13.5\%) showed malignant lesions. Postmenopausal women were 18 cases; 16 cases (89\%) showed malignant lesions and only two cases (11\%) had benign lesions (Fig. 2, Table 2). Out of the 112 lesions, 36 (32.1\%) were GI-RADS 2, 32 (28.6\%) GI-RADS 3, 13 (11.6\%) GI-RADS 4, and 31 (27.7\%) GI-RADS 5 (Fig. 3, Table 3).

The prevalence of malignant lesions by histopathology was 33/112 (29.5\%). According to the ultrasound findings, malignant lesions showed thick wall (wall thickness was $4-7 \mathrm{~mm}$, mean $4.16 \mathrm{~mm}$ ) in $57.6 \%$, compared to only $7.4 \%$ in benign lesions $(p<0.05)$. Benign lesions showed no septa in $59 \%$ of the lesions and thin septa (less than $3 \mathrm{~mm}$ ) in $41 \%$ of lesions. Also, the diagnosis of malignant lesions associated significantly with thicker septa, papillary projections, and the presence of solid areas: $21.2 \%, 9.1 \%$, and $33.4 \%$ in malignant lesions versus $0 \%, 0 \%$, and $7.6 \%$ in benign lesions, respectively $(p<0.05)$. In addition, all malignant lesions showed Doppler flow. The flow in the malignant lesions was central in 32 lesions (97\%) affecting the solid parts, papillae or septa.

Lesions classified as GI-RADS 2 (Fig. 4) included 35 cases presenting with 36 lesions. All lesions were either functional or complicated functional cysts. On follow-up ultrasound, 24 lesions showed complete resolution and 12 lesions revealed stationary course. All cases were premenopausal except one postmenopausal case. The lesions showed thin wall, no papillary projections, and thin septa in 10 lesions.

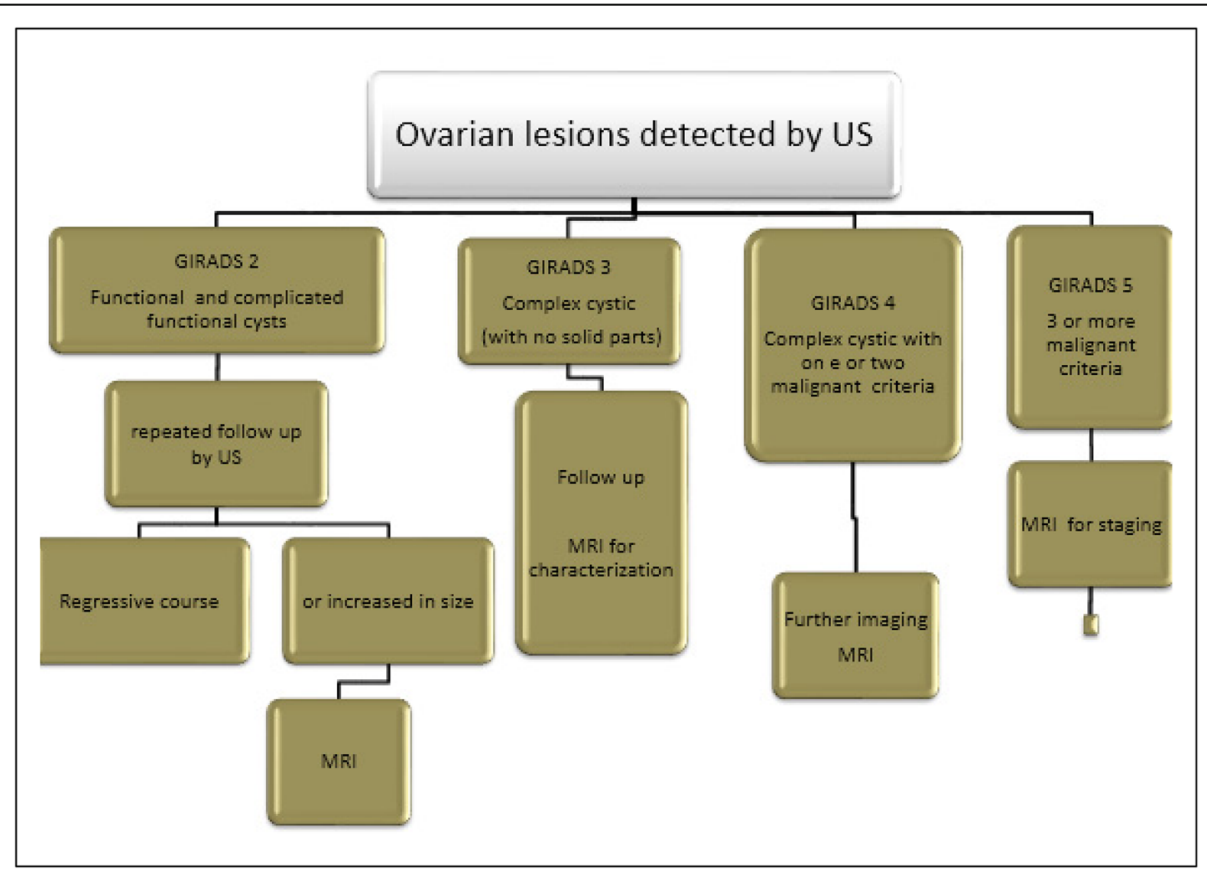

Fig. 1 Suggested algorithm for the diagnosis of ovarian lesions 


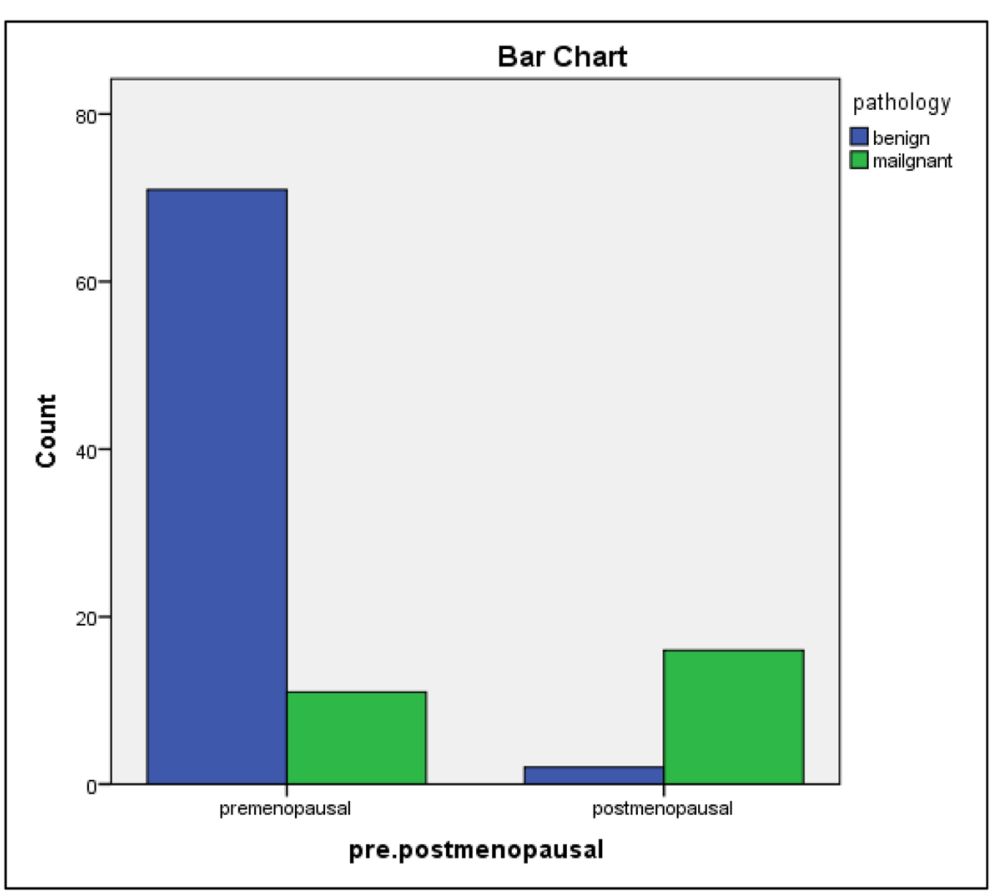

Fig. 2 Correlation between age whether pre- or postmenopausal and the pathology of the lesions

GI-RADS 3 lesions included 17 neoplastic lesions, 6 tubo-ovarian lesions, 7 endometriomas, and 2 paraovarian cysts. The neoplastic lesions included 16 benign lesions and one malignant lesion (false-negative case) diagnosed by histopathology as serous cystadenocarcinoma (48-yearold patient). By transvaginal ultrasound, the lesion was well defined, thin walled, thin septa, no papillary projections, no solid areas (Fig. 5). It showed low-level internal echoes and mild peripheral vascularity. Surgical treatment was done for 19 cases (22 lesions), while 9 cases (10 lesions) showed improvement and spontaneous resolution on follow-up ultrasound examination (Fig. 6).

Lesions categorized as GI-RADS 4 were 13 lesions, 3 of them (23\%) proved to be malignant neoplastic lesions (immature ovarian teratoma and primary ovarian carcinoma). The rest of the lesions included five benign neoplastic lesions (serous cystadenomas and one teratoma), two pedunculated subserous fibroid, one endometrioma, and two tubo-ovarian complex. Regarding the benign ovarian neoplastic lesions

Table 2 GIRADS classification and patient's age whether pre or postmenopausal

\begin{tabular}{llll}
\hline & Premenpausal & postmenopausal & Total \\
\hline GIRADS 2 & 34 & 1 & 35 \\
GIRADS 3 & 28 & 0 & 28 \\
GIRADS 4 & 11 & 0 & 11 \\
GIRADS 5 & 9 & 17 & 26 \\
Total & 82 & 18 & 100 \\
\hline
\end{tabular}

misclassified in this group, we found thick walls in two lesions, suspected solid component in two lesions, and associated ascites in one lesion. The tuboovarian lesions misclassified in this group; one of them was tubo-ovarian abscess with thick wall and suspected solid component and the other patient had haemorrhagic tubo-ovarian complex which appeared multilocular with increased peripheral vascularity and suspected solid component, the patient gave history of bleeding tendency (Fig.7). The endometrioma lesion misclassified in this group showed atypical ultrasound appearance. All cases of this group were managed by surgery except two cases including the endometrioma and the haemorrhagic tubo-ovarian complex lesion. They showed improvement on medical treatment. Pelvic MRI was recommended for patients under conservative management, for better lesion characterization.

GI-RADS 5 included 31 lesions, 29 of them were malignant: Primary ovarian malignancies were found in 16 lesions, 3 lesions diagnosed as ovarian lymphoma (Fig. 8), and secondary malignancy in 10 lesions. Two lesions were false positive; one diagnosed as benign fibrothecoma on histopathology (Fig. 9). The other case was ovarian torsion-detorsion with high vascularity (Fig. 10), showing regression on follow-up.

To calculate the overall diagnostic accuracy of GI-RADS classification among the 112 lesions, the ultrasound findings were compared to histopathological references in 55 cases (63 lesions) and the follow-up findings of ultrasound 


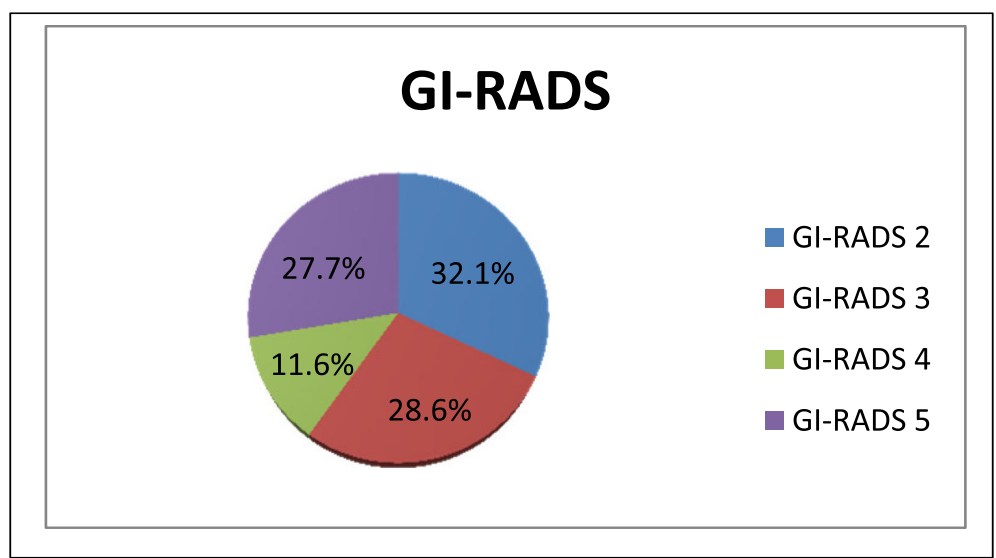

Fig. 3 Distribution of the adnexal lesions by GI-RADS

examination in the other 45 cases (49 lesions). The sensitivity of GI-RADS stood at $97 \%$, specificity $84.8 \%$, positive predictive value $68.1 \%$, negative predictive value $98.5 \%$, and accuracy $88.4 \%$ (Table 4 ).

The total number of ovarian neoplastic lesions in our study was 55 lesions. All neoplastic lesions were managed by surgery. They included 33 malignant and 22 benign lesions. Histopathological classification $[17,18]$ was done (Table 5). The diagnostic accuracy of GI-RADS classification in diagnosis of ovarian neoplastic lesions is shown in Table 6.

\section{Discussion}

Reporting of the precise diagnosis of adnexal masses is an important issue in clinical practice, as inaccurate diagnosis might lead to unnecessary examinations and surgeries, and appropriate diagnosis improves the communication between the medical team and leads to better outcome [19, 20].

This study detected the clinical usefulness of ultrasound reporting system GI-RADS in the diagnosis of

Table 3 Distribution of the adnexal lesions by GI-RADS

\begin{tabular}{llllll}
\hline Adnexal lesions & Gl- & Gl- & Gl- & Gl- & Total \\
& 2 & 3 & 4 & 5 & \\
\hline $\begin{array}{l}\text { RAnctional and complicated } \\
\text { cysts }\end{array}$ & 36 & 0 & 0 & 0 & 36 \\
Ovarian torsion & 0 & 0 & 0 & 1 & 1 \\
Neoplastic lesions & 0 & 17 & 8 & 30 & 55 \\
Paraovarian cysts & 0 & 2 & 0 & 0 & 2 \\
Endometriomas & 0 & 7 & 1 & 0 & 8 \\
$\begin{array}{l}\text { Tubo-ovarian lesions } \\
\text { Pedunculated subserous }\end{array}$ & 0 & 6 & 2 & 0 & 8 \\
uterine fibroid & 0 & 0 & 2 & 0 & 2 \\
Total & 36 & 32 & 13 & 31 & 112 \\
\hline
\end{tabular}

adnexal masses after evaluating different criteria. The prevalence of malignant lesions was 29.5\%. Malignant lesions were more likely to show thick walls, thick septa, papillary projections, solid areas, and central blood flow. Postmenopausal patients had higher incidence of malignant lesions while in premenopausal patients, most lesions were benign. In our study, we had one postmenopausal patient classified as GIRADS 2, the lesion showed regressive course on follow-up ultrasound examination. Postmenopausal women may present with functional or simple ovarian cysts that could be detected by ultrasound examination, saving surgical intervention and unnecessary imaging studies. The presence of functional or simple ovarian cysts in such age group was due to the residual ovarian activity [21].

Our results came in consistency with the study done by Zhang and colleagues who conducted a retrospective study over 263 adnexal masses and concluded that thick wall, solid papillary projections, solid areas, and central blood flow were associated with malignant lesions. The sensitivity and specificity of GI-RADS were $96.4 \%$ and $84.3 \%$, respectively [22].

The 112 lesions were distributed by GI-RADS classification as the following: 36 (32.1\%) GI-RADS 2, 32 (28.6\%) GI-RADS 3, 13 (11.6\%) GI-RADS 4, and 31 (27.7\%) GI-RADS 5. The ovarian neoplastic lesions represented 55 lesions $(49 \%)$ of the total number of the detected lesions in our study. The diagnostic accuracy of GI-RADS classification in the assessment of ovarian neoplastic lesions according to ultrasound findings and guided by the histopathological classification were $97 \%$, $73 \%, 84 \%, 94 \%$, and $87 \%$ for sensitivity, specificity, PPV, NPV, and accuracy, respectively.

The present study included one false-negative and twelve false-positive lesions. The false-negative lesion was misclassified as GI-RADS 3, as there was no 


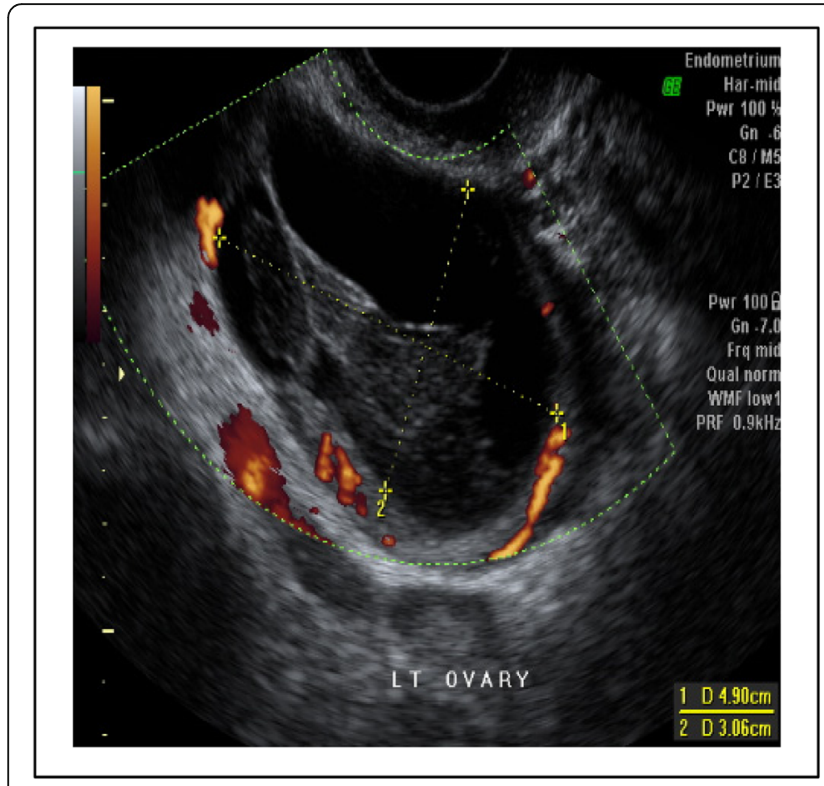

A.

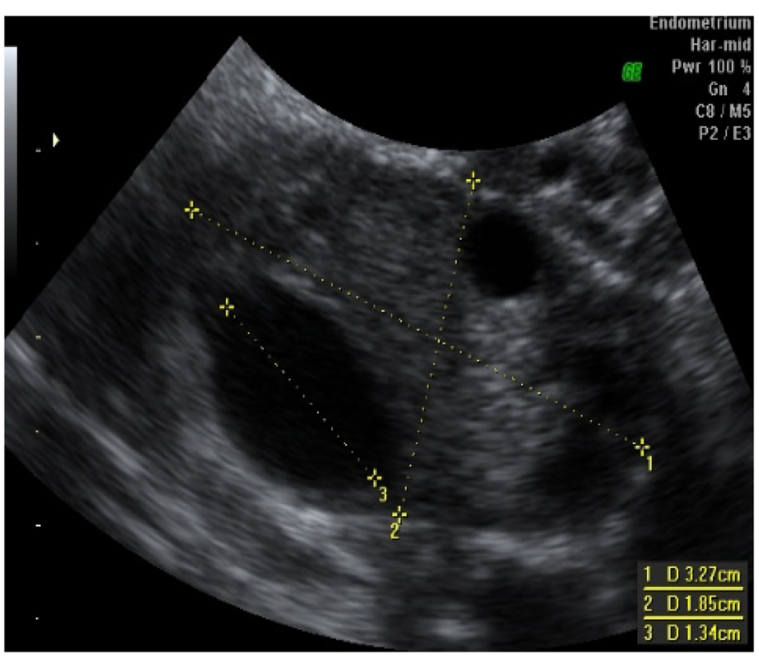

B.

Fig. 4 GI-RADS 2 complex cyst. a The echogenic non-vascular part. b The regressive course on follow-up

ultrasound findings suggestive of malignancy. The histopathological diagnosis was serous cystadenocarcinoma. This was similar to a study done by Migda et al, who found two malignant lesions out of 119 lesions categorized as GI-RADS 1-3 [23].

The twelve false-positive lesions included 6 benign ovarian neoplastic lesions, one case with ovarian torsiondetorsion, one endometrioma lesion with atypical ultrasound findings, two tubo-ovarian complex, and two

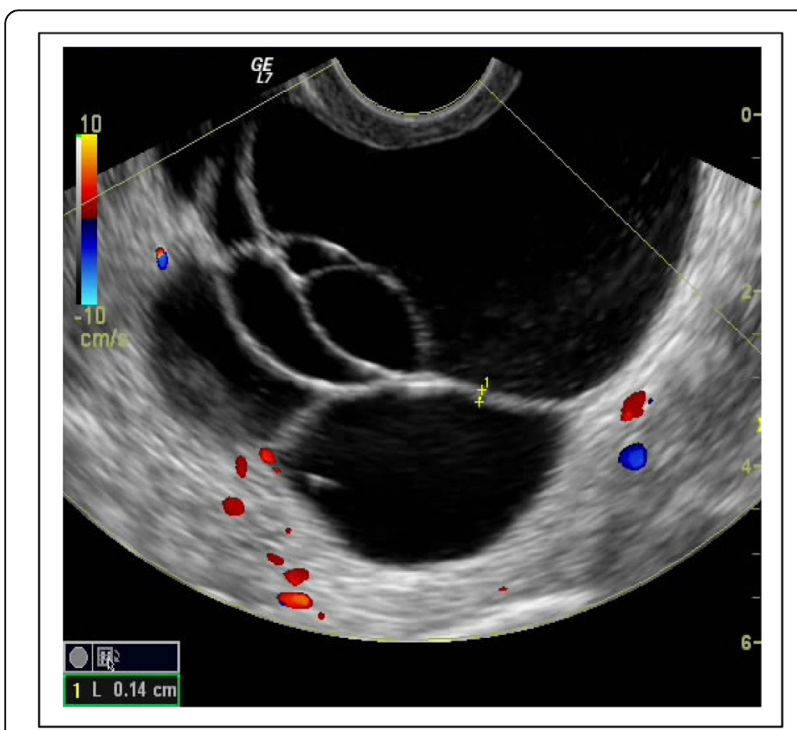

Fig. 5 GI-RADS 3, false-negative case diagnosed by histopathology as serous cystadenocarcinoma. By transvaginal ultrasound, well defined, thin walled, thin septa, no papillary projections, no solid areas pedunculated subserous fibroids. In case of ovarian torsion-detorsion misclassified as GI-RADS 5, the patient presented with vague clinical picture and there was no definite history of acute pain. The ultrasound examination revealed a large highly vascular ovary, but on follow-up ultrasound examination, there was regression in the ovarian size and vascularity.

The endometrioma lesion was misclassified as GIRADS 4 in our study due to the atypical ultrasound findings: multilocular cystic lesion with suspected solid component. Regarding the two tubo-ovarian lesions, there was no recent history of pain or fever; borderline ovarian tumours were considered as a differential diagnosis. This agrees with the previous studies suggesting that about $50 \%$ of female patients presenting with chronic tuboovarian abscesses may have normal body temperature and nonspecific clinical symptoms including vaginal discharge, abnormal vaginal bleeding, or mild abdominal pain. These clinical signs and symptoms may mimic borderline or malignant ovarian tumours. In such cases, further assessment by pelvic MRI examination for better characterization of the lesions is recommended [24, 25].

In the current study regarding the diagnosis of neoplastic lesions, we had 22 benign lesions and 33 malignant lesions. The GI-RADS classification rates in GI-RADS 4 were 5 benign neoplastic lesions (false positive) and 3 malignant lesions. In GI-RADS 5, there were 29 malignant lesions and one benign neoplastic lesion (false positive). The specificity was $73 \%$. This agrees to a great extent with the study done by Migda et al. [23], who reported 45 benign and 50 malignant lesions. In the GI-RADS 5 group, there 


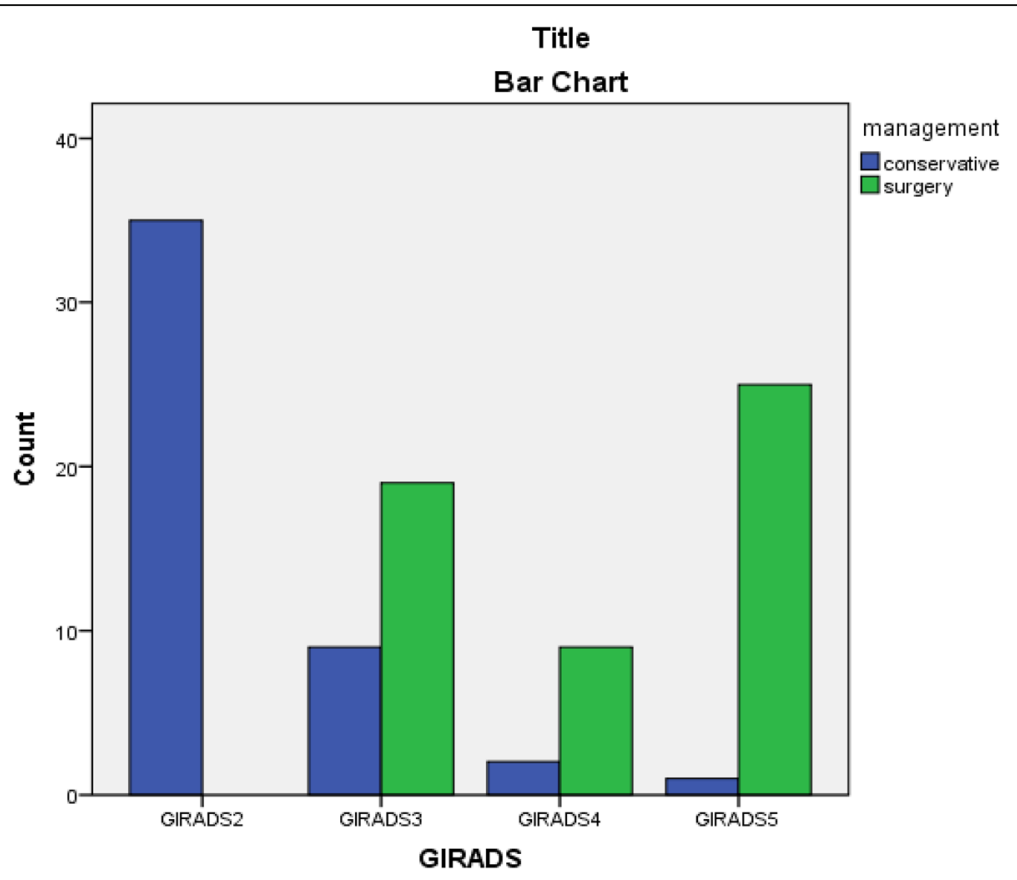

Fig. 6 Management of the adnexal lesions guided by GI-RADS classification

were only malignant lesions while in the GI-RADS 4 group, there were about 45 benign lesions (false-positive lesions) and the specificity of the study was $72 \%$. The false-positive lesion misclassified as GI-RADS 5 in our study due to the solid appearance and the increased vascularity of the lesion was diagnosed as fibrothecoma benign by histopathology.
The present study also agrees with a study done by Amor et al. [12], who reported that no malignant lesion was classified as GI-RADS 2 and one malignant lesion (false negative) was misclassified as GI-RADS 3. Amor et al. [12] found that the prevalence of malignant lesions was $26 \%$. Their lesions were classified by GI-RADS as follows: 92 (21\%) GI-RADS 2, 184 (43\%) GI-RADS 3, 40
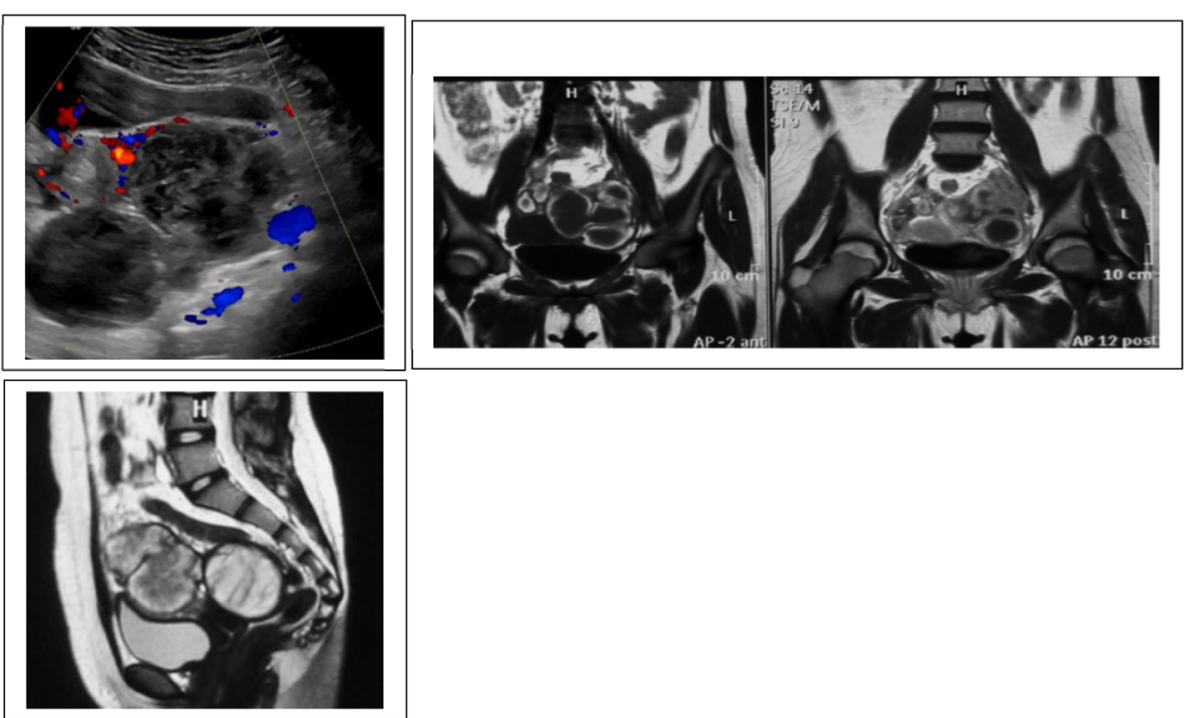

Fig. 7 Haemorrhagic tubo-ovarian complex lesion. Female patient 17 years old presented by menorrhagia. a US image revealed left complex adnexal mass with thick septa, only peripheral vascularity was seen. b-d MRI T1 pre- and postcontrast, T2 revealed haemorrhagic tubo-ovarian complex. On clinical and laboratory assessment, bleeding tendency was detected with final diagnosis of idiopathic thrombocystopenic purpura 


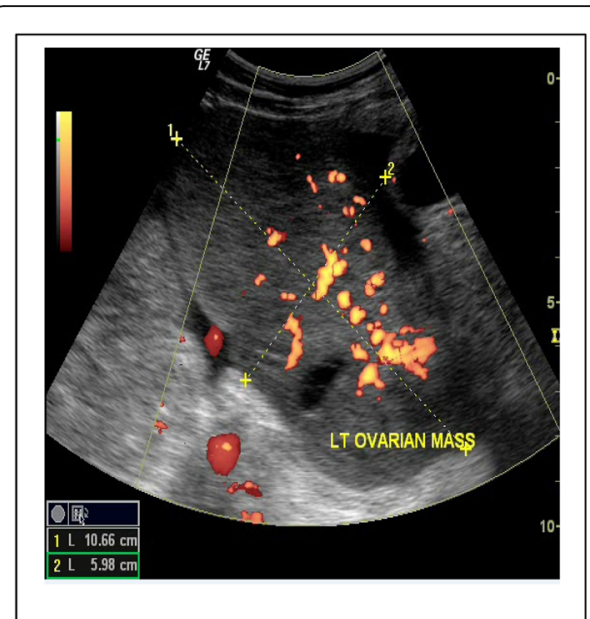

A.

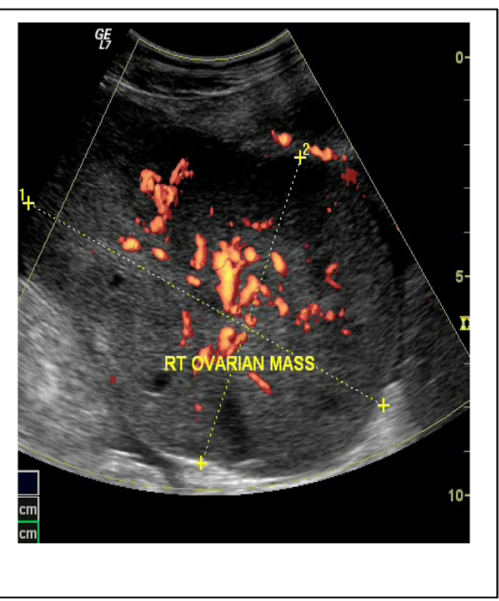

B.

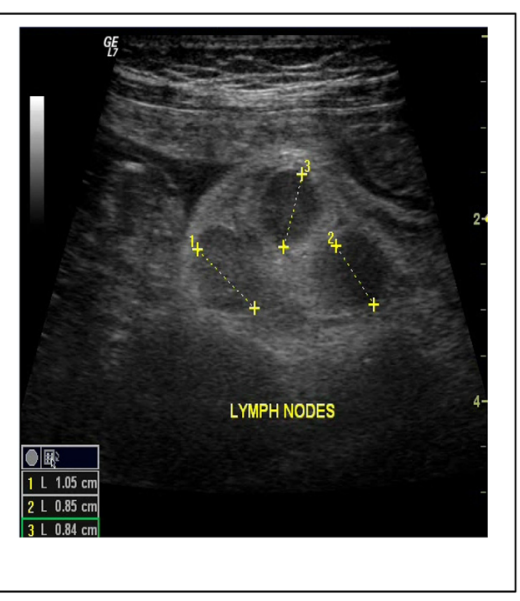

C.

Fig. 8 Gl-RADS 5. A 30-year-old female patient presenting by ascites, subacute intestinal obstruction. On abdominal and pelvic US, a and $\mathbf{b}$ revealed bilateral enlarged ovaries with hypoechoic stroma, preserved follicles, high central vascularity on power Doppler, and multiple mesenteric lymph nodes. Bilateral secondary ovarian lymphoma was the pathological diagnosis

(9\%) GI-RADS 4, and 116 (27\%) GI-RADS 5, and the sensitivity of the system was $99.1 \%$, specificity $85.9 \%$, PPV 71.1\%, and NPV 99.6\%.

It is also worth pointing out that GI-RADS reporting system will be of great use as it enhances communication between radiologists and gynaecologists for better diagnosis and proper management of the patients presenting with adnexal lesions based on clinical and ultrasound morphological characteristics of the lesion [12].

\section{Conclusion}

We concluded that GI-RADS reporting system performed well with high sensitivity and adequate specificity; it could

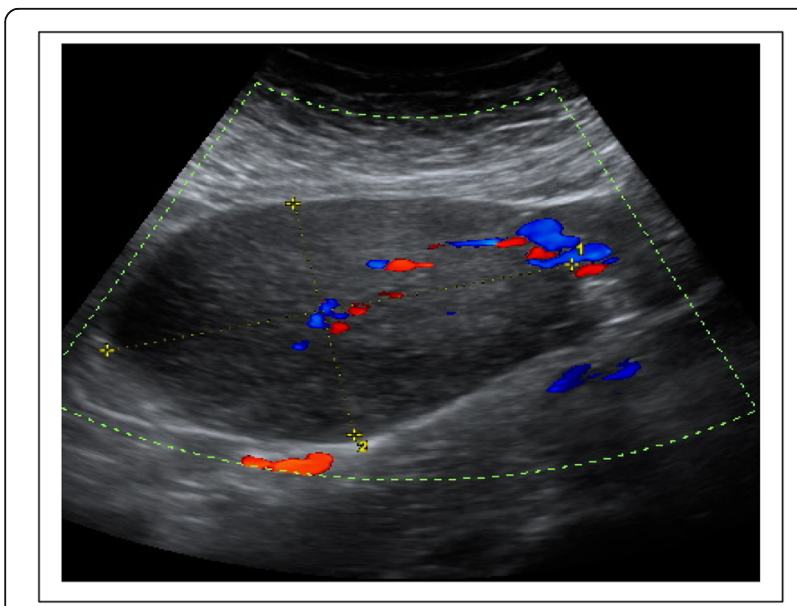

Fig. 9 A 60-year-old female patient showing an enlarged hypoechoic left ovary with central vascularity, hence was classified as GI-RADS 5. Panhystrectomy was done, and pathology revealed benign fibrothecoma accurately diagnose 49 (43.8\%) benign lesions as GI-RADS $2-3$, so saved the patient's further imaging and surgical intervention. The increased number of benign lesions misclassified as GI-RADS 4 required additional markers to improve the specificity in GI-RADS classification.

\section{Recommendation}

Our recommendations to improve the diagnostic performance of GI-RADS scoring system include patient demographic data, proper history taking, and Doppler

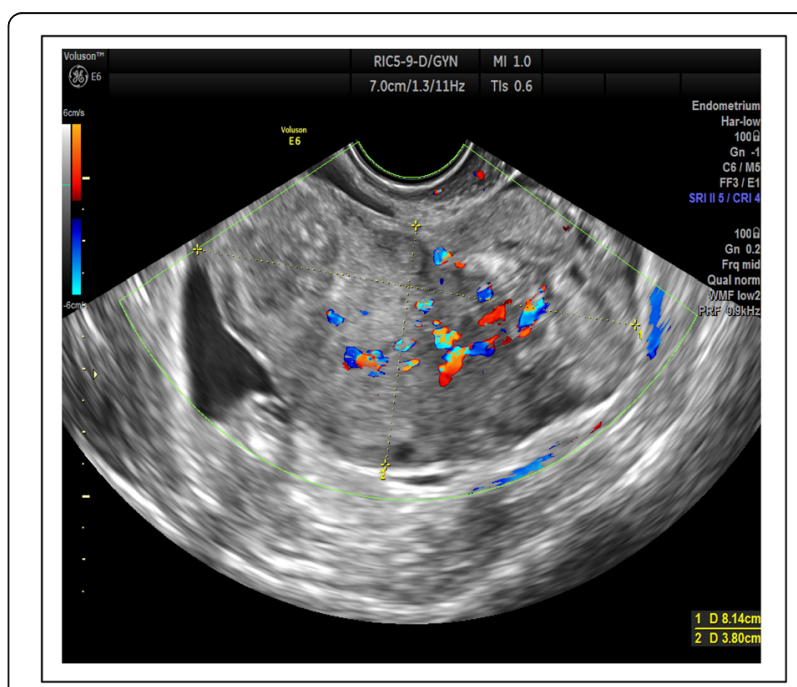

Fig. 10 GI-RADS 5. A 28-year-old female patient presented by vague abdominal pain and abdominal distention. On US and colour Doppler, an enlarged left ovary was seen with high central vascularity, lack of arborization mimicking malignant circulation. CA 125 was normal. On follow-up US, regression of the mass took place. Final diagnosis of ovarian torsion-detorsion was done 
Table 4 The overall diagnostic performance of GI-RADS classification in diagnosis of the detected (112 lesions) ovarian lesions

\begin{tabular}{lll}
\hline $\begin{array}{lll}\text { Ultrasound } \\
\text { findings }\end{array}$ & Final diagnosis & Malignant $n=33$ \\
\cline { 2 - 3 } GI-RADS 2-3 & $67(\mathrm{TN})$ & 1 (FN) \\
GI-RADS 4-5 & $12(\mathrm{FP})$ & $32(\mathrm{TP})$ \\
Sensitivity & $97 \%$ & \\
Specificity & $84.8 \%$ & \\
PPV & $72.7 \%$ & \\
NPV & $98.5 \%$ & \\
Accuracy & $88.4 \%$ & \\
\hline
\end{tabular}

Table 6 The diagnostic accuracy of GI-RADS classification in diagnosis of neoplastic ovarian lesions

\begin{tabular}{lll}
\hline \multirow{2}{*}{$\begin{array}{l}\text { Ultrasound } \\
\text { findings }\end{array}$} & \multicolumn{2}{l}{ Final diagnosis } \\
\cline { 2 - 3 } & Benign $n=22$ & Malignant $n=33$ \\
\hline GI-RADS 3 & $16(\mathrm{TN})$ & 1 (FN) \\
GI-RADS 4-5 & $6(\mathrm{FP})$ & $32(\mathrm{TP})$ \\
Sensitivity & $97 \%$ & \\
Specificity & $73 \%$ & \\
PPV & $84 \%$ & \\
NPV & $94 \%$ & \\
Accuracy & $87 \%$ &
\end{tabular}

Table 5 Correlation between histopathological classification and GI-RADS reporting system [17, 18]

\begin{tabular}{|c|c|c|c|c|}
\hline \multirow{2}{*}{ Histopathologiacal classification } & \multicolumn{2}{|c|}{ GIRADS classification } & \multirow[b]{2}{*}{ GIRADS 5} & \multirow{2}{*}{ Total } \\
\hline & GIRADS 3 & GIRADS 4 & & \\
\hline \multirow{3}{*}{\multicolumn{4}{|c|}{$\begin{array}{l}\text { 1-Primary ovarian tumours: } \\
\text { 1.1 Surface epithelial stromal ovarian tumours. } \\
1.1 .1 \text { ovarian serous tumours }\end{array}$}} & \\
\hline & & & & \\
\hline & & & & \\
\hline Ovarian serous cystadenoma & 4 & 4 & 0 & 8 \\
\hline Ovarian serous cystadenocarcinoma & 1 & 0 & 0 & 1 \\
\hline \multicolumn{5}{|l|}{ 1.1.2 Ovarian mucinous tumours } \\
\hline Ovarian mucinous cystadenoma & 1 & 0 & 0 & $\mathbf{1}$ \\
\hline Ovarian mucinous cystadenocarcinoma & 0 & 0 & 2 & 2 \\
\hline 1.1.3 Ovarian carcinoma & 0 & 2 & 11 & 13 \\
\hline 1.1.4 malignant Brenner's tumour & 0 & 0 & 1 & $\mathbf{1}$ \\
\hline \multirow{2}{*}{\multicolumn{5}{|c|}{$\begin{array}{l}\text { 1.2 Germ cell ovarian tumours } \\
1.2 .1 \text { Ovarian teratoma }\end{array}$}} \\
\hline & & & & \\
\hline Mature ovarian (cystic) teratoma & 7 & 1 & 0 & 8 \\
\hline Immature ovarian teratoma & 0 & 1 & 1 & 2 \\
\hline 1.2.2 Primary ovarian choriocarcinoma & 0 & 0 & 1 & 1 \\
\hline \multicolumn{5}{|l|}{ 1.3 Sex cord/ stromal ovarian tumours } \\
\hline 1.3.1 Ovarian fibrothecoma & & & & \\
\hline Ovarian fibroma & 3 & 0 & 0 & 3 \\
\hline Ovarian fibrothecoma & 1 & 0 & 1 & 2 \\
\hline \multicolumn{5}{|l|}{ 2-Others } \\
\hline 2.1 Ovarian lymphyma & $\mathbf{0}$ & $\mathbf{0}$ & 3 & 5 \\
\hline 2.2 Metastatic lesions & $\mathbf{0}$ & $\mathbf{0}$ & 10 & 10 \\
\hline Total & 17 & 8 & 30 & 55 \\
\hline
\end{tabular}


study. Further classification of GI-RADS 4 category into subgroups according to the degree of malignancy depending on the examiner's impression and patient age may be required. This will decrease inconclusive ultrasound results, the number of misclassified cases, and the number of cases requiring additional imaging study by pelvic MRI.

\section{Abbreviations}

GI-RADS: Gynaecologic Imaging Reporting and Data System;

IOTA: International Ovarian Tumour Analysis; NPV: Negative predictive value; PPV: Positive predictive value; TVS: Transvaginal sonography

\section{Acknowledgements}

Not applicable.

\section{Authors' contributions}

SA carried out the statistical analysis, data collection, image analysis, drafting, and editing of the paper. SH shared in the image analysis, interpretation, and manuscript editing. MS participated in the data collection and clinical assessment. All contributing authors have read and approved the manuscript.

\section{Funding}

No funding resources.

\section{Availability of data and materials}

All data are available at the corresponding author who has the authority to respond if there is any query.

\section{Ethics approval and consent to participate}

The study was approved from the ethical committee of Faculty of Medicine Beni Suef University (FWA00015574). Data were collected after obtaining informed written consent of all cases.

\section{Consent for publication}

All patients included in this research gave written informed consent to publish the data contained within this study.

\section{Competing interests}

The authors declare that they have no competing interests.

\section{Author details}

${ }^{1}$ Department of Radiology, Faculty of Medicine, Beni-Suef University, Beni Suef, Egypt. ${ }^{2}$ Department of Radiology, Faculty of Medicine, Cairo University, Giza, Egypt. ${ }^{3}$ Department of Obstetrics and Gynecology, Faculty of Medicine, Beni-Suef University, Beni Suef, Egypt.

Received: 28 January 2020 Accepted: 26 February 2020

Published online: 20 April 2020

\section{References}

1. Castillo G, Alcázar JL, Jurado M (2004) Natural history of sonographically detected simple unilocular adnexal cysts in asymptomatic postmenopausal women. Gynecol Oncol. 92:965-969

2. Alcázar JL, Castillo G, Jurado M, García GL (2005) Is expectant management of sonographically benign adnexal cysts an option in selected asymptomatic premenopausal women? Hum Reprod. 20:3231-3234

3. Ferlay J, Soerjomataram I, Ervik M, Dikshit R, Eser S, Mathers C et al (2013) GLOBOCAN 2012 v1.0, Cancer incidence and mortality worldwide: IARC CancerBase No. 11. Lyon, France, International Agency for Research on Cancer http://globocan.iarc.fr

4. Ibrahim AS, Khaled HM, Mikhail NNH, Baraka H, Kamel H (2014) Cancer incidence in Egypt: results of the National Population-Based Cancer Registry Program. Journal of Cancer Epidemiology 2014:18 pages

5. Howlader N, Noone AM, Krapcho M, Neyman N, Aminou R, Waldron W et al. SEER Cancer Statistics Review, 1975-2008, National Cancer Institute. Bethesda, MD, http://seer.cancer.gov/csr/1975_2008/, based on November 2010 SEER data submission, posted to the SEER web site, 2011.

6. Practice ACOG (2007) Bulletin. Management of adnexal masses. American College of Obstetricians and Gynecologists. Obstet Gynecol 110:201-214
7. Valentin L (1999) Pattern recognition of pelvic masses by gray-scale ultrasound imaging: the contribution of Doppler ultrasound. Ultrasound Obstet Gynecol 14:338-347

8. Granberg S, Wikland M, Jansson I (1989) Macroscopic characterization of ovarian tumors and the relation to the histological diagnosis: criteria to be used for ultrasound evaluation. Gynecol Oncol 35:139-144

9. Alcázar JL, Mercé LT, Laparte C, Jurado M, López-García G. A new scoring system to differentiate benign from malignant adnexal masses. Am J Obstet Gynecol 2003; 188: 685-692.

10. Alcázar JL, Errasti T, Laparte C, Jurado M, López-García G. Assessment of a new logistic model in the preoperative evaluation of adnexal masses. J Ultrasound Med 2001; 20: 841-848.

11. Timmerman D, Verrelst $H$, Bourne TH, DeMoor B, Collins WP, Vergote I et al (1999) Artificial neural network models for the preoperative discrimination between malignant and benign adnexal masses. Ultrasound Obstet Gynecol 13:17-25

12. Amor F, Alcázar JL, Vaccaro H, León M, Iturra A (2011) GI-RADS reporting system for ultrasound evaluation of adnexal masses in clinical practice: a prospective multicenter study. Ultrasound in Obstetrics and Gynecology. 38:450-455

13. Amor F, Vaccaro H, Alcázar JL, León M, Craig JM, Martinez J. Gynecologic imaging reporting and data system: a new proposal for classifying adnexal masses on the basis of sonographic findings. J Ultrasound Med 2009; 28: 285-291.

14. Spinelli C, Pucci V, Strambi S, Piccolo RL, Martin A, Messineo A (2015) Treatment of ovarian lesions in children and adolescents: a retrospective study of 130 cases. Pediatr Hematol Oncol. 32:199-206

15. Watanabe E, Tanaka K, Takeda N, Takeda N, Takayasu H, Yokota K, Watanabe M (2013) Surgical technique to prevent spillage of cyst fluid during operation for cystic ovarian tumors. Pediatr Surg Int. 29:645-649

16. Woo YL, Kyrgiou M, Bryant A, Everett T, Dickinson HO (2012) Centralisation of services for gynaecological cancers, a Cochrane systematic review. Gynecol Oncol. 126:286-290

17. Bazot M, Cortez A, Sananes S et. al. Imaging of pure primary ovarian choriocarcinoma. AJR Am J Roentgenol. 2004;182 (6): 1603-4. AJR Am J Roentgenol (full text) - Pubmed citation

18. Jeong YY, Outwater EK, Kang HK. Imaging evaluation of ovarian masses. Radiographics. 2000; 20 (5): 1445-70. Radiographics (full text) - Pubmed citation

19. Brown DL, Dudiak KM, Laing FC (2010) Adnexal masses: US characterization and reporting. Radiology. 254:342-354

20. Levine D, Brown DL, Andreotti RF, Benacerraf B, Benson CB, Brewster WR et al (2010) Management of asymptomatic ovarian and other adnexal cysts imaged at US: Society of Radiologists in Ultrasound Consensus Conference Statement. Radiology 256:943-954

21. Greenlee RT, Kessel B, Williams CR, Riley TL, Ragard LR, Hartge P, Buys SS, Partridge $\mathrm{EE}$, Reding DJ. Prevalence, incidence, and natural history of simple ovarian cysts among women $>55$ years old in a large cancer screening trial. Am J Obstet Gynecol. 2010 Apr;202(4):373.e1-9. doi: 10.1016/j.ajog.2009.11. 029. Epub 2010 Jan

22. Zhang T, Li F, Liu J, Zhang S (2017) Diagnostic performance of the Gynecology Imaging Reporting and Data System for malignant adnexal masses. Int J Gynaecol Obstet. 137(3):325-331

23. Migda M, Kierszk M, Migda M, Malenczyk M. Evaluation of GIRADS system in assessment of adnexal masses malignancy risk in clinical practice retrospective study. Ultrasound in Obstet Gynecol 2016; 48 (Suppl. 1): P12.05.

24. Rakheja $\mathrm{R}$, Makis W, Hickeson M. Bilateral tubo-ovarian abscess mimics ovarian cancer on MRI and (18)F-FDG PET/CT. Nucl Med Mol Imaging. 2011 45: 223- 228. doi: 10.1007/s13139-011-0089-5PMID: 24900009

25. Velcani A, Conklin P, Specht N (2010) Sonographic features of tubo-ovarian abscess mimicking an endometrioma and review of cystic adnexal masses. J Radiol Case Rep. 4:9-17

\section{Publisher's Note}

Springer Nature remains neutral with regard to jurisdictional claims in published maps and institutional affiliations. 